\section{Look harder}

\section{A neuroscience initiative aims to end the invisibility of female scientists at conferences.}

$\mathrm{R}$ elatively few women make it to top academic positions in science - and there begins the vicious circle of invisibility. Women aren't available as mentors for aspiring young scientists. They aren't there when journalists call for someone to provide a quick scientific opinion. And they are apparently not thought of when conference organizers put together lists of speakers to invite to meetings, says a group of frustrated neuroscientists trying to do something practical about the problem.

Fed up with attending meetings where most invited speakers are men, even when there are plenty of competent women to choose from, the group has created BiasWatchNeuro to bring a more systematic approach to monitoring and challenging the gender balance of academic conferences. Have a look at it: it's an eye-opener.

As successful neuroscientists themselves, the women (and a few men) behind the name-and-shame initiative know about bias-free sampling. They would like to see gender parity on speaker lists, to counter some of the many biases that hold women back. But they lobby most insistently for the minimum decency: that the percentage of women invited to speak at a particular meeting is at least equal to the base rate of women in its field.

They have worked out the base rate for neuroscience as a whole $-24 \%$ - from looking at the proportion of women in the faculties of top US universities. They use other information sources to work out the base rate for each subdiscipline - sometimes by looking at attendance lists of important meetings, more often by turning to the US National Institutes of Health (NIH) list of investigatorinitiated grants, which can be searched with keywords, and simply counting up the number of female and male grant-winners. Particular subdisciplines may have other ways of working out the base rate.

Since starting in August last year, they have analysed more than
90 conferences. Two meetings last month show what makes the group angry. One was on memory mechanisms in health and disease, a subject that the NIH grant-winner list suggests has a base rate of $42 \%$ women. It mustered only 2 female invited speakers in a line-up of 17 - just $12 \%$. The other was on tools and protocols for handling big neuroscience data, a subject in computational neuroscience, which has a low base rate of just $17-20 \%$. The organizers managed to find no women at all to

"In our scientific
society, women
tend to be
invisible."
include among the 14 invited speakers.

Why does this happen? It is almost certainly not down to a conscious desire to exclude women. But we all unthinkingly develop biases that are shaped by the society we operate in. In our scientific society, women tend to be invisible. It's that vicious circle. Can initiatives like BiasWatchNeuro help to end it? Simply bringing the issue into open discussion in such clearly scientific terms helps a lot. The prestigious US Computational and Systems Neuroscience meeting Cosyne used to be male-dominated but, thanks to vocal complaints in the past few years, its gender ratio of invited speakers is now routinely above the field's base rate. It is one of the shining examples on BiasWatchNeuro. Its equivalent in Europe, the Bernstein Conferences, has been exposed: last year, it mustered only one female invited speaker. Whether because it felt shamed, or because BiasWatchNeuro has given women confidence to insist, it has $42 \%$ female speakers this year - well over the field's base rate.

Conference organizers should not feel that they have done their duty if they invite a top woman scientist who declines. The most successful women in science get inundated with invitations, but there will always be other successful women to choose from, and identifying them has been made easy. Anne's List (created by computational neuroscientist Anne Churchland at Cold Spring Harbor Laboratory in New York) groups female neuroscientists easily into topic and seniority level. In Europe, AcademiaNet identifies women across scientific disciplines.

The creators of BiasWatchNeuro chose the name - even though the simpler BiasWatch.com domain was available - because they hope that other scientists will get together to organize BiasWatchAnotherdiscipline.com. Nature urges you to do so. Female scientists, you have nothing to lose but your invisibility.

\section{Sharp practice}

\section{Monkeys can make tool-like objects, but that doesn't mean they know what they're doing.}

$\mathrm{T}$ echnology is often a tale of seamless acquisition and refinement of skills - from rocks banged together, and bows and arrows, to steam engines and integrated circuits. But the appearance of artefacts is a different thing from their makers' intentions - if any.

As researchers show in a study published online in Nature this week (T. Proffitt et al. Nature http://dx.doi.org/nature20012; 2016), capuchin monkeys (Sapajus libidinosus) from the Serra da Capivara National Park in Brazil smash rocks in a way that produces sharp-edged stone flakes; were these flakes associated with an early Stone Age site, they might be regarded as intentionally produced. Indeed, progress in Stone Age technology is sometimes measured in terms of an increase in the number of sharp edges that can be coaxed from a given amount of raw material. This, of course, presupposes that producing flakes is, in fact, the intention. But capuchins, having created stone flakes, let them lie.

Why the monkeys go to all that effort remains a mystery. However, the researchers observed that about half of the monkeys sniffed or licked the broken surfaces afterwards, suggesting that they break rocks to extract mineral supplements in a conveniently powdered form.
Other monkeys bash rocks together, but the capuchins are the only wild, non-human primates known to do so with the seeming intention of breaking them. Chimps sometimes break rocks by mistake, but even when taught to bang rocks together with intent, bonobos don't create anything that resembles what is found in the hominin record.

Recognizing the earliest stone tools for what they are is not always easy, but certain features mark artefacts as the product of intent. These include the 'conchoidal' flaking that leaves a distinctive percussion mark; the production of several flakes from a single core, and the use of specific patterns of flake removal. Such features distinguish artefacts from geofacts - that is, rocks broken by natural processes, rather than objects made by non-human animals - but they say little or nothing about how the artefacts might have been used. As the capuchin example shows, the intent of the makers of the earliest artefacts can be hard to discern. The producers of the earliest stone tools to be generally recognized as such (S. Harmand et al. Nature 521, 310-315; 2015) lived 3.3 million years ago, and were very different from modern humans.

The capuchin study should also dampen ideas that the human hand, with its precision grip, together with advanced hand-eye coordination, must necessarily have been evolutionary products or prerequisites of technology. Capuchins break rocks without the benefit of either.

In the end, the activity of banging rocks together should be seen as precisely that, and not as the first, proleptic step towards the stars. The ape-man at the start of 2001: A Space Odyssey that throws a bone in the air that becomes a space station was, after all, a modern human in a gorilla suit. 